Rev. Biol. Trop., 47(3): 593-596, 1999

www.ucr.ac.cr www.ots.ac.cr www.ots.duke.edu

\title{
Trematodiasis en algunos peces del lago de Cuitzeo, Michoacán, México
}

\author{
Ma. del Carmen Guzmán-Cornejo y Luis García-Prieto \\ Laboratorio de Helmintología, Instituto de Biología, UNAM, A.P. 70-153; C.P. 04510, México, D.F.; fax 55500164 , \\ correo electrónico: gprieto@mail.ibiologia.unam.mx
}

Recibido 16-XI-1998. Corregido 3-II-1999. Aceptado 9-II-1999.

\begin{abstract}
In September, 1995 and January, 1996, six species of fishes from Lake Cuitzeo, Michoacan, Mexico, were collected (Alloophorus robustus $\mathrm{n}=30$, Goodea atripinnis $\mathrm{n}=30$, Xenotoca variata $\mathrm{n}=41$, Chirostoma jordani $\mathrm{n}=30$, Carassius auratus $\mathrm{n}=30$ and Oreochromis aureus $\mathrm{n}=30$ ), to establish the infection levels reached by Posthodiplostomum minimum, Clinostomum complanatum and Diplostomum (Tylodelphys) sp., in their hosts. Infection caused by P. minimum metacercariae was the most important helminthiasis considering the number of hosts they parasite (five of six), and the high levels of abundance of the infection; we suggest that fish preferences and the generalist character of the trematode are the causes that determine this infection. Finally, the role of goodeids and atherinids in transmission of infections in the lake is confirmed; the former being the main group mantaining such infections.
\end{abstract}

Key words: Mexico, fresh water fishes, Clinostomiasis, Diplostomiasis, Posthodiplotomiasis.

Las infecciones ocasionadas por metacercarias de Posthodiplostomum minimum (MacCallum, 1921) Dubois, 1936, Clinostomum complanatum (Rudolphi, 1814) Braun, 1899 y Diplostomum (Tylodelphys) Diesing, 1850, se encuentran entre las principales helmintiasis que afectan a los peces dulceacuícolas de México, donde $P$. minimum ha sido registrado como parásito de 25 especies de peces, mientras que $C$. complanatum se ha recolectado en 33 y D. (Tylodelphys) sp., en cuatro (Pérez-Ponce de León et al. 1996). A partir de lo anterior, en el presente trabajo se analizó una parte de la fauna ictiológica del Lago de Cuitzeo, Michoacán, México, $\left(19^{\circ} 58^{\prime} \mathrm{N}\right.$, y $101^{\circ} 08^{\prime} \mathrm{W}$, a 1820 $\mathrm{msnm}$ ), perteneciente a la cuenca del Río Lerma-Santiago, con el fin de establecer los niveles de infección que registran estas especies de helmintos en sus hospederos.

Los muestreos fueron desarrollados en septiembre de 1995 y enero de 1996, recolectándose las seis especies de peces mas abun- dantes y distribuidas en el lago, de acuerdo con Medina et al. (1992), mediante redes "agalleras" y "chinchorros": Alloophorus robustus (Bean, 1892) $(\mathrm{n}=30)$, Carassius auratus (Linneo) $(\mathrm{n}=30)$, Chirostoma jordani Woolman, $1894(\mathrm{n}=30)$, Goodea atripinnis Jordan, 1880 $(\mathrm{n}=30)$, Oreochromis aureus (Steindachner) $(\mathrm{n}=30)$ y Xenotoca variata (Bean, 1887) $(\mathrm{n}=41)$. De manera complementaria, se recolectaron algunas aves ictiófagas utilizando arma de fuego, con el fin de obtener los estadios adultos de los tremátodos encontrados en los peces, para efectuar su determinación taxonómica: Casmerodius albus (Linneo, 1758) $(\mathrm{n}=$ dos), Fulica americana Gmelin, $1789(\mathrm{n}=$ tres), Nycticorax nycticorax (Linneo, 1758) $(\mathrm{n}=\mathrm{uno})$ y Anas americana Gmelin, $1789(\mathrm{n}=$ siete). La revisión de los hospederos, la recolecta de helmintos y su posterior procesamiento, se realizaron con base en la metodología referida por Pérez-Ponce de León (1995). Material de referencia depositado en la Colección 
Nacional de Helmintos del Instituto de Biología de la UNAM (CNHE), con los números de catálogo: P. minimum: 3000,3003 a 3006; D. (Tylodelphys) sp.; 2998, 3002; C. complanatum: 2996, 2997, 2999, 3001. La caracterización de las infecciones se realizó con base en los parámetros prevalencia y abundancia, definidos por Margolis et al. (1982).

La identificación de las metacercarias registradas en los peces del lago, fue realizada a partir de la correlación entre sus rasgos y los de los adultos encontrados en las aves. De esta forma, del intestino de $N$. nycticorax se obtuvieron 20 gusanos adultos asignados a la especie P. minimum, de acuerdo con Pérez-Ponce de León (1995). En esta misma especie de hospedero, se recolectó un ejemplar de C. complanatum, alojado en la cavidad bucal. Su identificación se realizó con base en el estudio de la sistemática del género efectuado por Ukoli (1966); finalmente, el tremátodo recolectado en el intestino de la "garza" C. albus, se determinó como D. (Tylodelphys) sp. siguiendo el criterio de Dubois (1970); no obstante, el mal estado del material, impidió asignarlo a alguna de las especies descritas para el mismo, incluyendo a $D$. (Tylodelphys) americana (Dubois, 1936) Dubois, 1937, especie previamente registrada por León (1992) en la cuenca del río Lerma-Santiago.

Posthodiplostomum minimum parasitó cinco de las seis especies de peces (Cuadro 1). La infección fue mayor en la familia Goodeidae, particularmente en A. robustus (KruskalWallis, $\mathrm{F}=30.82, \mathrm{p}=0.00$ ). Lo anterior puede atribuirse a que estos peces habitan en aguas lénticas, poco profundas, con maleza acuática (Chacón 1980), donde también se distribuyen los primeros hospederos intermediarios del parásito (caracoles), aumentando la probabilidad de encuentro entre éste y sus hospederos; en el caso de A. robustus, además, los resultados sugieren una mayor susceptibilidad del pez hacia el helminto, aspecto que ratifica lo señalado por autores como Pérez-Ponce de León (1992), Meléndez y Rosas (1995), y Rojas et al. (1997), quienes encontraron que este hospedero es uno de los más importantes para el helminto, entre los peces del Lago de Pátzcuaro, Michoacán, en términos de prevalencia y abundancia. En el caso de $C$. complanatum, cuyas metacercarias también parasitaron de manera diferencial a las tres especies de godeidos analizadas (Kruskal-Wallis, $\mathrm{F}=51.71$, $\mathrm{p}=0.00$ ), es probable que se presente una situación similar, al coexistir estrechamente hospederos y parásitos, debido a las preferencias de hábitat del pez y a la alta susceptibilidad a la infección por parte de los miembros de esta familia, como lo demuestra el hecho de que en la cuenca del Río Lerma-Santiago, los godeidos han constituido el principal grupo de hospederos para el helminto (Pérez-Ponce de León et al. 1996); por el contrario, los niveles alcanzados por $D$. (Tylodelphys) sp., fueron mas elevados en $C$. jordani que en G. atripinnis $(\mathrm{Zcal}=1.6559>\mathrm{Z} 0.05(1)=1.6449)$; la primera especie de hospedero pertenece a la familia Atherinidae, a la cual se encuentra generalmente asociado el parásito en la región lacustre del estado de Michoacán en la que se localiza Cuitzeo (Meléndez y Rosas 1995, Pérez-Ponce de León et al. 1996), siendo la primera ocasión que se le registra en peces de una familia distinta (Goodeidae) en la zona; no obstante, los reducidos valores de prevalencia y abundancia con que se presentó en $G$. atripinnis, sugieren la naturaleza accidental de la infección.

Los niveles de prevalencia y abundancia alcanzados por $P$. minimum, lo establecen como el tremátodo más importante para los peces del lago. Esto puede ser explicado con base en las características biológicas del helminto, particularmente la reducida especificidad hospedatoria que exhibe para sus hospederos intermediarios (peces) y definitivos (aves) en la región (Ramos 1994) y los elevados parámetros de infección que registra en los mismos, lo que en conjunto, posibilita que su ciclo biológico se complete regularmente, manteniendo el flujo de formas infectivas espacial y temporalmente, de manera similar a lo referido por Rojas et al. (1997), al analizar la estructura de las comunidades de helmintos en godeidos del Lago de Pátzcuaro. 


\section{CUADRO 1}

Trematodiasis registradas en seis especies de peces del Lago de Cuitzeo, Michoacán

\begin{tabular}{|c|c|c|c|c|c|c|}
\hline \multirow[b]{2}{*}{ Hospedero } & \multicolumn{2}{|c|}{ P. minimum } & \multicolumn{2}{|c|}{ D. (Tylodelphys) sp. } & \multicolumn{2}{|c|}{ C. complanatum } \\
\hline & $\%$ & $\mathrm{Ab}$ & $\%$ & $\mathrm{Ab}$ & $\%$ & $\mathrm{Ab}$ \\
\hline Goodeidae & & & & & & \\
\hline A. robustus & 93.3 & 53.3 & 0 & 0 & 90 & 29.5 \\
\hline G. atripinnis & 86.6 & 23 & 6.6 & 0.06 & 13.2 & 0.63 \\
\hline$X$. variata & 80.4 & 21 & 0 & 0 & 26.8 & 1.2 \\
\hline Atherinidae & & & & & & \\
\hline $\begin{array}{l}\text { C. jordani } \\
\text { Cyprinidae }\end{array}$ & 66.6 & 6.9 & 30 & 1.5 & 0 & 0 \\
\hline C. auratus 1 & 0 & 0 & 0 & 0 & 0 & 0 \\
\hline Cichlidae & & & & & & \\
\hline O. aureus 1 & 6.6 & 0.06 & 0 & 0 & 0 & 0 \\
\hline
\end{tabular}

\section{AGRADECIMIENTOS}

A Gerardo Pérez-Ponce de León, por la lectura y crítica al manuscrito original y a Agustín Jiménez Ruíz por la asesoría para el análisis estadístico de los datos.

\section{REFERENCIAS}

Chacón , A. 1980. Contribución al conocimiento de la ecología y composición de la ictiofauna del Lago de Cuitzeo, Michoacán. Tesis de Licenciatura, Universidad Michoacana de San Nicolás de Hidalgo, Morelia, Michoacán, México.

Dubois, G. 1970. Synopsis des Strigeata et Diplostomatidae (Trematoda). Mem. Soc. Neuchatel Sci. Nat. 10: 259-727.

León, V. 1992. Fauna helmintológica de algunos vertebrados acuáticos de la Ciénaga de Lerma, México. Anales Inst. Biol. Univ. Nac. Autón. Méx. Ser. Zool. 63: 151-153.

Margolis, L., G. Esch, J. Holmes, A. Kuris \& G. Schad. 1982. The use of ecological terms in Parasitology (report of ad hoc commitee of the American Society of Parasitologists). J. Parasitol. 68: 131-133.

Medina, M., R. Moncayo \& M. Gallegos. 1992. Estado actual de la ictiofauna de la ribera este del Lago de Cuitzeo, Michoacán. III Congreso Nacional de Ictiología, Sociedad Ictiológica Mexicana, Oaxtepec, México. 8p.
Meléndez, C. \& M. Rosas G. 1995. Algunos aspectos ecológicos de las helmintiasis que afectan a las especies de peces endémicas del Lago de Pátzcuaro, Michoacán, México. Tesis de Licenciatura, Universidad Nacional Autónoma de México, México D. F., México.

Pérez-Ponce de León G. 1992. Sistemática del género Posthodiplostomum Dubois, 1936 y algunos aspectos epizootiológicos de la Postodiplostomiasis en el lago de Pátzcuaro, Michoacán, México. Tesis de Doctorado, Universidad Nacional Autónoma de México, México D. F. México.

Pérez-Ponce de León, G. 1995. Host induced morphological variability in adult Posthodiplostomum minimum. J. Parasitol. 81: 818-820.

Pérez-Ponce de León, G., L. García, D. Osorio \& V. León. 1996. Listados Faunísticos de México. VI. Helmintos parásitos de peces de aguas continentales de México. Instituto de Biología, UNAM, México D. F., México. 100 p.

Ramos R., P. 1994. Composición de la comunidad de helmintos del tubo digestivo de tres especies de "garzas" (Ciconiiformes: Ardeidae) del lago de Pátzcuaro, Michoacán, México. Tesis de Maestría, Universidad Nacional Autónoma de México, México, D. F., México.

Rojas, E., G., Pérez-Ponce de León \& L. García. 1997. Helminth community structure of some freshwater fishes from Patzcuaro, Michoacan, Mexico. Trop. Ecol. 38: 129-131

Ukoli, F. A. 1966. On Clinostomum tilapiae n. sp., and C. phalacrocoracis Dubois, 1931, from Ghana and a discussion of the systematics of the genus Clinostomum Leidy, 1856. J. Helminthol. 40: 187-214. 